\title{
MS12-P06 | BUILDING THE PROTEIN STRUCTURE-SPECIFIC SIDE-CHAIN ROTAMER LIBRARIES
}

Grybauskas, Algirdas (Vilnius University Institute of Biotechnology, Vilnius, LTU); Gražulis, Saulius (Vilnius University Institute of Biotechnology, Vilnius, LTU)

Identifying the probable positions of the protein side-chains is one of the protein modeling steps that can greatly improve the prediction of protein-ligand, protein-protein interactions. Most of the strategies predicting the sidechain conformations use pre-determined angles, also called rotamer libraries, that are usually generated from the subset of high-quality protein structures from PDB. Grouping the amino acids by their type and calculating the frequencies of the most occurring dihedral angles in that group are then performed. Depending on the library, groups can be further divided by the structure of the main-chain atoms, solvent accessibility or other criteria.

Although these methods are fast, they tend to be too generalized and ignore basic assumptions about the geometry and surroundings of the target protein that can result in inaccuracies when predicting the possible sidechain atom positions; Hence we propose the approach that takes into account both of these terms by scanning through the conformationally restricted side-chain positions and generating dihedral angle libraries specific to the target proteins. The method avoids the drawbacks of lacking conformations due to unusual or rare protein structures. The core principle of the approach is a limited movement of side-chains imposed by the fixed number of degrees of freedom. Combinatorial explosion is avoided by using dead-end elimination and cell-list neighbor search while calculating potential energy.

Building such dynamic libraries could increase the likelihood of detecting rare rotamers and reveal the broader picture on the impact of side-chain positions. 